\title{
Mechanism of Near-Field Raman Enhancement in One-Dimensional Systems
}

\author{
L. G. Cançado, ${ }^{1}$ A. Jorio, ${ }^{2}$ A. Ismach, ${ }^{3}$ E. Joselevich, ${ }^{3}$ A. Hartschuh, ${ }^{4}$ and L. Novotny ${ }^{1, *}$ \\ ${ }^{1}$ The Institute of Optics, University of Rochester, Rochester, New York 14627, USA \\ ${ }^{2}$ Departamento de Física, Universidade Federal de Minas Gerais, Belo Horizonte, MG, 30123-970, Brazil \\ ${ }^{3}$ Department of Materials and Interfaces, Weizmann Institute of Science, Rehovot 76100, Israel \\ ${ }^{4}$ Department Chemie und Biochemie and CeNS, Ludwig-Maximilians-Universität München, 81377 München, Germany
}

(Received 24 June 2009; revised manuscript received 7 October 2009; published 26 October 2009)

We develop a theory of near-field Raman enhancement in one-dimensional systems, and report supporting experimental results for carbon nanotubes. The enhancement is established by a laserirradiated nanoplasmonic structure acting as an optical antenna. The near-field Raman intensity is inversely proportional to the 10th power of the separation between the enhancing structure and the one-dimensional system. Experimental data obtained from single-wall carbon nanotubes indicate that the Raman enhancement process is not significantly influenced by the specific phonon eigenvector, and is mainly defined by the properties of the nanoplasmonic structure.

DOI: 10.1103/PhysRevLett.103.186101

PACS numbers: $68.37 . \mathrm{Uv}, 78.30 . \mathrm{Na}$

The photophysics of one-dimensional systems such as semiconductor nanorods and carbon nanotubes has developed to the point of studying local defects that distort the system, renormalize energies and act as trap states [1]. Near-field Raman scattering, which employs a metal tip as an optical antenna, affords the spatial resolution needed to identify spectral variations associated with defects at the nanometer scale [1-3]. Historically, near-field Raman spectroscopy remained marginal until recently due to technical limitations [4]. The experimental achievement of the tip-enhanced Raman spectroscopy (TERS) revolutionized the field [4], and the technique is now being used by groups worldwide [5].

In order to be able to quantify experimental data and to relate measurements to intrinsic material's properties we here provide an analytical theory of local field enhancement in one-dimensional (1D) systems. Our theory predicts that the near-field Raman intensity is inversely proportional to the 10th power of the tip-sample distance. Supporting the theory, experimental results of near-field Raman measurements performed on single-wall carbon nanotubes (SWNT) are presented. The experimental data show that all Raman peak intensities exhibit the same tipsample distance dependence and that there are negligible spectral variations.

Figure 1 shows the experimental configuration and the coordinates used in the theoretical analysis. The electric field $\mathbf{E}$ near the laser-irradiated gold tip is axially symmetric [6] and interacts locally with a SWNT at frequency $\omega$. The induced dipole $\mathbf{p}$ per unit length at the Raman frequency $\omega_{s}$ and at location $\mathbf{r}^{\prime}$ can be represented as

$$
\mathbf{p}\left(\mathbf{r}^{\prime}, \omega_{s}\right)=\overleftrightarrow{\boldsymbol{\alpha}}^{R}\left(\mathbf{r}^{\prime} ; \omega_{s}, \omega\right) \mathbf{E}_{\mathrm{tot}}\left(\mathbf{r}^{\prime}-\mathbf{r} ; \omega\right),
$$

where $\mathbf{r}$ denotes the position of the center of the tip apex, $\overleftrightarrow{\boldsymbol{\alpha}}^{R}$ is the Raman polarizability (per unit length), and $\mathbf{E}_{\text {tot }}$ is the total electric field interacting with the electron density at $\mathbf{r}^{\prime}$ in the carbon nanotube.
In this analysis we will concentrate on the radial breathing mode (RBM) and the $G^{+}$and $G^{-}$modes, which are all one-phonon Raman processes involving vibrations belonging to the totally symmetric $A_{1 g}$ irreducible representation, i.e., they do not break the tube symmetry [7]. For all three modes we can represent the Raman polarizabilty tensor $\overleftrightarrow{\boldsymbol{\alpha}}^{R}$ as [7]

$$
\overleftrightarrow{\boldsymbol{\alpha}}_{q}^{R}=\left[\begin{array}{ccc}
\alpha_{\perp, q}^{R} & 0 & 0 \\
0 & \alpha_{\perp, q}^{R} & 0 \\
0 & 0 & \alpha_{\|, q}^{R}
\end{array}\right], \quad q \in\left\{\mathrm{RBM}, G^{+}, G^{-}\right\}
$$

The field $\mathbf{E}_{\text {tot }}$ in Eq. (1) is the sum of the external driving field $\mathbf{E}$ and screening fields due to neighboring charges, and can be represented as [8]

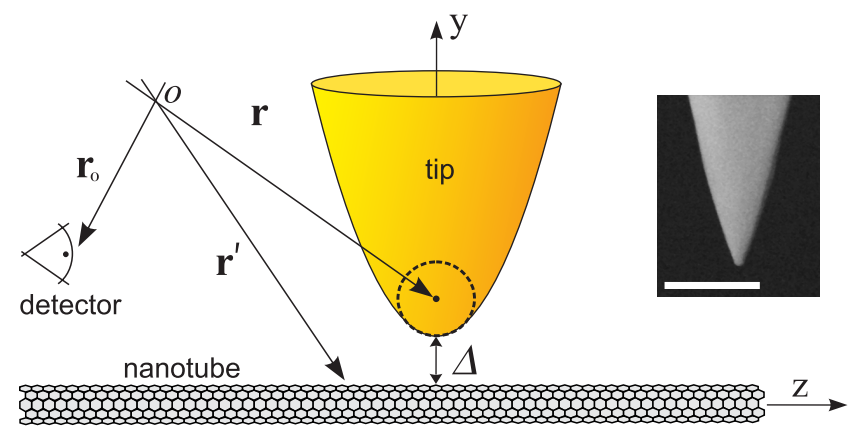

FIG. 1 (color online). Schematics for the spatially resolved Raman scattering of carbon nanotubes. The position vectors $\mathbf{r}$, $\mathbf{r}^{\prime}$, and $\mathbf{r}_{o}$ denote the location of the center of the tip apex, the Raman dipole moment $\mathbf{p}$, and the detector, respectively. $O$ indicates the origin of the coordinate system. The dashed circumference represents a small sphere of radius $r_{\text {tip }}$ centered at the tip apex, and $\Delta$ is the distance between the tip and the nanotube. The inset depicts an SEM image of the gold tip used, where the scale bar denotes $200 \mathrm{~nm}$. 


$$
\mathbf{E}_{\text {tot }}=\overleftrightarrow{\mathbf{d}}(\omega) \mathbf{E}=\left[\begin{array}{ccc}
\left(1+\alpha_{\perp} / 4 \pi \varepsilon_{o} \rho_{\text {tube }}^{2}\right)^{-1} & 0 & 0 \\
0 & \left(1+\alpha_{\perp} / 4 \pi \varepsilon_{o} \rho_{\text {tube }}^{2}\right)^{-1} & 0 \\
0 & 0 & 1
\end{array}\right] \mathbf{E}
$$

Here, $\varepsilon_{o}$ is the permittivity of vacuum, $\rho_{\text {tube }}$ is the nanotube radius, and $\alpha_{\perp}$ is the unscreened nanotube polarizability in transverse direction. The depolarization matrix $\overleftrightarrow{\mathbf{d}}(\omega)$ indicates that, transverse to the tube axis, the driving field is reduced relative to the incident field.

The external driving field $\mathbf{E}$ in Eq. (3) corresponds to the superposition of the incident laser field $\mathbf{E}_{o}$ and the localized field generated by the gold tip acting as an optical antenna. Close to the tip apex, the external driving field $\mathbf{E}\left(\mathbf{r}^{\prime} ; \omega\right)$ resembles the field of an induced dipole $\boldsymbol{\mu}$ located at the center of a small sphere of radius $\rho_{\text {tip }}$ (location $\mathbf{r}$ in Fig. 1) and can be approximated as [6]

$$
\mathbf{E}\left(\mathbf{r}^{\prime}, \omega\right) \approx \mathbf{E}_{o}\left(\mathbf{r}^{\prime}, \omega\right)+\frac{\omega^{2}}{\varepsilon_{o} c^{2}} \overleftrightarrow{\mathbf{G}}^{o}\left(\mathbf{r}, \mathbf{r}^{\prime} ; \omega\right) \overleftrightarrow{\boldsymbol{\alpha}}_{\mathrm{tip}}(\omega) \mathbf{E}_{o}(\mathbf{r}, \omega)
$$

Here, $\overleftrightarrow{\mathbf{G}}^{o}$ is the free-space dyadic Green's function in the absence of a tip [9], $\overleftrightarrow{\boldsymbol{\alpha}}_{\text {tip }}$ is the tip's polarizability, and $c$ is the vacuum speed of light. In what follows, we must neglect the incident laser field $\mathbf{E}_{o}\left(\mathbf{r}^{\prime}, \omega\right)$ in Eq. (4) because the local field due to the metal tip is generally much stronger. Adhering to the coordinates defined in Fig. 1, $\overleftrightarrow{\boldsymbol{\alpha}}_{\text {tip }}$ can be represented as [10]

$$
\overleftrightarrow{\boldsymbol{\alpha}}_{\text {tip }}=4 \pi \varepsilon_{o} \rho_{\text {tip }}^{3}\left[\begin{array}{ccc}
(\varepsilon-1) /(\varepsilon+2) & 0 & 0 \\
0 & f_{e} / 2 & 0 \\
0 & 0 & (\varepsilon-1) /(\varepsilon+2)
\end{array}\right] .
$$

Here, $f_{e}$ is the complex field-enhancement factor, which depends on the tip material and geometry. Typical values are $f_{e} \approx-2.9+11.8 i[6]$. The transverse polarizability components correspond to the quasistatic polarizability of a small sphere (dashed circumference in Fig. 1) of radius $\rho_{\text {tip }}$ and dielectric constant $\varepsilon$. To summarize so far, the laser-irradiated metal tip generates a localized field $\mathbf{E}$, which gives rise to a screened Raman excitation field $\mathbf{E}_{\text {tot }}$, which in turn generates a Raman dipole $\mathbf{p}$ (per unit length) in the carbon nanotube.

The scattered field at the detector (location $\mathbf{r}_{o}$ in Fig. 1) with shifted frequency $\omega_{s}$ is the field generated by the tip's secondary sources induced by the Raman dipole distribution $\mathbf{p}\left(z^{\prime}, \omega_{s}\right)$. Similar to Eq. (4) we restrict these secondary sources to the leading dipole term $\mathbf{p}_{\text {tip }}\left(\mathbf{r}, \omega_{s}\right)$ and obtain for the recorded intensity of the Raman scattered field

$$
\mathbf{E}\left(\mathbf{r}_{o}, \omega_{s}\right) \propto \int_{-\infty}^{+\infty} d z^{\prime} \overleftrightarrow{\boldsymbol{\alpha}}_{\mathrm{tip}}\left(\omega_{s}\right) \overleftrightarrow{\mathbf{G}}^{o}\left(\mathbf{r}, z^{\prime} ; \omega_{s}\right) \mathbf{p}\left(z^{\prime}, \omega_{s}\right)
$$

The detected intensity is proportional to the spectral density, which is the ensemble average of the square modulus of the field given in Eq. (6), leading to $I_{\mathrm{NF}}\left(\mathbf{r}_{o}, \omega_{s}\right) \propto$ $\left\langle\mathbf{E}^{*}\left(\mathbf{r}_{o}, \omega_{s}\right) \cdot \mathbf{E}\left(\mathbf{r}_{o}, \omega_{s}\right)\right\rangle$. This relation requires the sub- stitution of the Raman dipole moment $\mathbf{p}$ defined by Eqs. (1)-(5).

To proceed we assume that the polarizability of the metal tip is much stronger along its axis, i.e., $f_{e} \gg$ $(\varepsilon-1) /(\varepsilon+2)$, a necessary condition for tip-enhanced Raman scattering (TERS). Using this condition we can write the integral sum in the Raman intensity $I_{\mathrm{NF}}$ as

$$
\begin{aligned}
I_{\mathrm{NF}}\left(\mathbf{r}_{o}, \omega_{s}\right) \propto & \rho_{\mathrm{tip}}^{12}\left|f_{e}(\omega) f_{e}\left(\omega_{s}\right)\right|^{2}\left|\mathbf{E}_{o}(\mathbf{r}, \omega) \cdot \hat{\mathbf{y}}\right|^{2} \\
& \times \mid \frac{\alpha_{\perp, q}^{R}\left(\omega_{s}, \omega\right)}{1+\alpha_{\perp}(\omega) / 4 \pi \varepsilon_{o} \rho_{\text {tube }}^{2}} \\
& \times \int_{-\infty}^{+\infty} d z^{\prime} G_{y y}^{o}\left(\mathbf{r}, z^{\prime} ; \omega\right) G_{y y}^{o}\left(\mathbf{r}, z^{\prime} ; \omega_{s}\right) \\
& +\alpha_{\|, q}^{R}\left(\omega_{s}, \omega\right) \\
& \times\left.\int_{-\infty}^{+\infty} d z^{\prime} G_{y z}^{o}\left(\mathbf{r}, z^{\prime} ; \omega\right) G_{y z}^{o}\left(\mathbf{r}, z^{\prime} ; \omega_{s}\right)\right|^{2},
\end{aligned}
$$

where $G_{i j}^{o}$ are tensor components of the free-space dyadic Green's function and $\hat{\mathbf{y}}$ is the unit vector in $y$. This equation corresponds to the fully coherent scattering (see discussion at the end of the Letter and also in Ref. [9]). Retaining only the near-field terms in $G_{i j}^{o}$ [9] the integrals can be readily integrated and we obtain

$$
\begin{aligned}
I_{\mathrm{NF}}\left(\mathbf{r}_{o}, \omega_{s}\right) \propto & \frac{\rho_{\text {tip }}^{12}\left|f_{e}(\omega) f_{e}\left(\omega_{s}\right)\right|^{2}}{\left(\Delta+\rho_{\text {tip }}\right)^{10}}\left|\mathbf{E}_{o}(\mathbf{r}, \omega) \cdot \hat{\mathbf{y}}\right|^{2} \\
& \times \mid \frac{\alpha_{\perp, q}^{R}\left(\omega_{s}, \omega\right)}{1+\alpha_{\perp}(\omega) / 4 \pi \varepsilon_{o} \rho_{\text {tube }}^{2}} \\
& +\left.\frac{15}{41} \alpha_{\|, q}^{R}\left(\omega_{s}, \omega\right)\right|^{2} .
\end{aligned}
$$

Here, $\Delta$ is the distance between tip and nanotube, as shown in Fig. 1. The one-dimensional integration over the length of the nanotube generates a distance dependence that scales with $\left(\Delta+\rho_{\text {tip }}\right)^{-10}$. Consistent with the theory of surface enhanced Raman scattering (SERS) the signal is enhanced by roughly the fourth power of the enhancement factor $f_{e}[11]$.

The appearance of the term proportional to $\alpha_{\perp}^{R}$ inside the square modulus in the second line of Eq. (8) has an important consequence for the near-field Raman scattering of carbon nanotubes. It is well established that optical transitions of the type $E_{\mu} \rightarrow E_{\mu}$ and $E_{\mu} \rightarrow E_{\mu \pm 1}$, where $\mu$ is the electronic state angular momentum, are allowed for light polarized parallel and perpendicular to the nanotube axis, respectively [7]. Because of the depolarization effect discussed in Eq. (3), far-field measurements usually render the longitudinal component of the polarizability [7], and only nanotubes whose $E_{\mu} \rightarrow E_{\mu}$ transition energies are in resonance with $\hbar \omega$ or $\hbar \omega_{s}$ can be detected. However, 
the above analysis demonstrates that the signal depends both on the transverse and the longitudinal Raman polarizabilities, since the small prefactor of $\alpha_{\|}^{R}$ in Eq. (8) increases the importance of $\alpha_{\perp}^{R}$ and the latter can no longer be neglected in near-field measurements. In this case, nanotubes whose optical transition energies $E_{\mu} \rightarrow E_{\mu \pm 1}$ are in resonance with $\hbar \omega$ or $\hbar \omega_{s}$ can also be studied by tipenhanced Raman spectroscopy.

We now compare the developed theory with near-field Raman measurements. The experimental details of tipenhanced Raman spectroscopy have been described before [2] and are not repeated here. Figure 2(a) shows a largescale confocal Raman image of a self-organized carbon nanotube serpentine [12]. The contrast (color scale) in the image represents the intensity of the graphitic (C-C stretch-

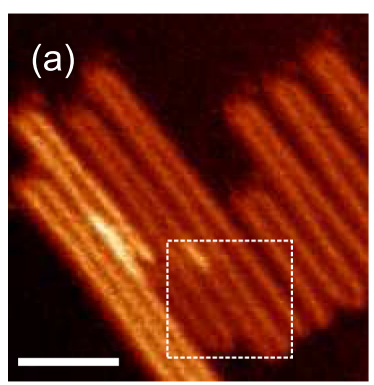

(c)

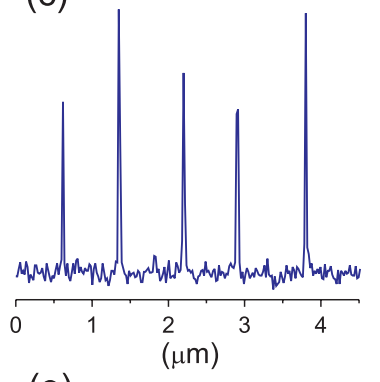

(e)

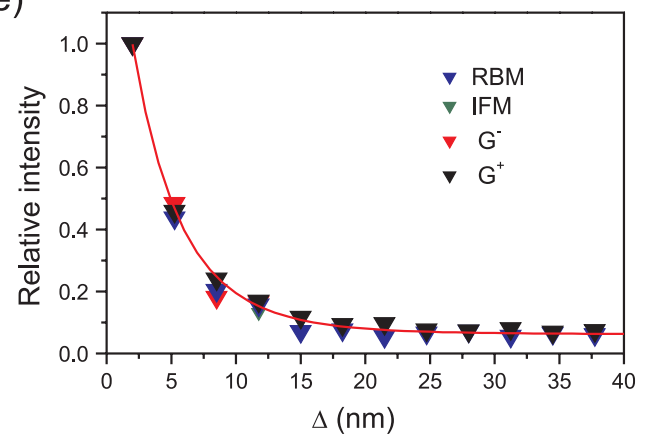

FIG. 2 (color online). (a) Confocal Raman image corresponding to the $G$ band intensity of a semiconducting nanotube. The scale bar denotes $4 \mu \mathrm{m}$. (b) Near-field Raman image corresponding to the $G$ band intensity recorded in the boxed area in panel (a). The scale bar denotes $800 \mathrm{~nm}$. (c) Intensity profile obtained along the dashed line in panel (b). (d) Far-field Raman spectrum (red or gray curve) and near-field Raman spectrum (black curve) recorded at the largest $(\sim 38 \mathrm{~nm})$ and smallest $(\sim 2 \mathrm{~nm})$ tip-sample separation, respectively. (e) Approach curves for the RBM, IFM, $G^{-}$, and $G^{+}$bands [7]. ing) $G$ band $\left(\sim 1580 \mathrm{~cm}^{-1}\right)$. Figure 2(b) shows a corresponding near-field Raman image acquired in the boxed area in 2(a). The resulting resolution of $25 \mathrm{~nm}$ is defined by the tip radius. A line-cut along the dashed line in image 2(b) is depicted in Fig. 2(c). It is evident that near-field imaging not only improves resolution but it also improves the signal-to-noise ratio.

Figure 2(d) shows the corresponding Raman spectra for the smallest $(\sim 2 \mathrm{~nm})$ and largest $(\sim 38 \mathrm{~nm})$ tip-sample separation. The two spectra are offset for clarity. The radial breathing mode $(\mathrm{RBM})$ frequency $\omega_{\mathrm{RBM}}=262 \mathrm{~cm}^{-1}$ identifies the sample as a semiconducting tube whose energy gap for the second $E_{\mu} \rightarrow E_{\mu}$ optical transition is in resonance with the incident laser energy (c.f. Kataura plot in Ref. [7]). The near-field spectrum also clearly features the intermediate frequency mode (IFM) occurring at $865 \mathrm{~cm}^{-1}$, and the $G^{-}$and $G^{+}$peaks occurring at $1545 \mathrm{~cm}^{-1}$ and $1585 \mathrm{~cm}^{-1}$, respectively [7]. Notice that the disorder-induced $D$ band $\left(\sim 1350 \mathrm{~cm}^{-1}\right)$ cannot be observed, indicating that the serpentine nanotube has a low defect density [7]. Within the experimental resolution, no differences in the lineshape and peak frequency of any spectral features were observed for the far-field and nearfield Raman spectra.

Figure 2(e) shows the intensities (integrated areas) of several Raman peaks as a function of the tip-sample separation $\Delta$. All Raman intensities are normalized with the corresponding values at shortest separation $(\Delta \sim 2 \mathrm{~nm})$. Considering that near-field (NF) and far-field (FF) components are always intermixed, the relative intensity of the scattered signal is given by the sum of these two contributions, i.e., $I / I_{\max }=\left(I_{\mathrm{FF}}+I_{\mathrm{NF}}\right) / I_{\max }$. Cross terms originating from the interference between FF and NF can be neglected. The ratio $I_{\max } / I_{\mathrm{FF}}$ corresponds to the maximum Raman enhancement factor $M$, which allows us to represent the relative intensity of the scattered signal as

$$
\frac{I}{I_{\max }}=\frac{1}{M}+\frac{C}{\left(\Delta+\rho_{\text {tip }}\right)^{10}},
$$

where the second term at the right-hand side is a simplification of Eq. (8). $M, C$, and $\rho_{\text {tip }}$ are parameters to be determined by fitting the experimental data. The red curve in Fig. 2(e) is a fit to the experimental data according to Eq. (9). It can be seen from Fig. 2(e) that our theoretical predictions are in good agreement with the experimental data. The fitting parameters obtained were $M=16, C=$ $4.5 \times 10^{15} \mathrm{~nm}^{10}$, and $\rho_{\text {tip }}=35 \mathrm{~nm}$. All modes present the same distance dependence, that is, within the resolution of our measurements, all phonon modes get enhanced by the same factor.

We have repeated our measurements on nanotubes of other chiralities and found very similar behavior. There was no significant difference between metallic and semiconducting nanotubes and for tubes that exhibit a resonance with the first or higher $E_{\mu} \rightarrow E_{\mu}$ optical transitions. For example, the fitting parameters according to Eq. (9) for 
a metallic nanotube turned out to be $M=16, C=4.8 \times$ $10^{15} \mathrm{~nm}^{10}$, and $\rho_{\text {tip }}=35 \mathrm{~nm}$. The values for $M$ and $\rho_{\text {tip }}$ are exactly the same as those that were derived for the semiconducting tube shown in Fig. 2. Because we used the same gold tip in all measurements we conclude that the maximum enhancement $M$ in a near-field Raman experiment is defined by the tip properties and is not influenced by the tube structure. Moreover, in spite of the fact that our theoretical analysis was restricted to a one-phonon Raman processes involving vibrational modes with $A_{1 g}$ symmetry, our experiments show that the theoretical analysis is also valid for two-phonon Raman processes such as 2 RBM and IFM. This is an indication that the Raman enhancement process is governed by the numerator of Eq. (8), i.e., by the fourth power of the field-enhancement factor.

The value for the tip radius obtained by fitting the data according to Eq. (9) is considerably larger than the spatial resolution of the near-field Raman images [c.f. Fig. 2(b)]. In fact, the intensity profile shown in Fig. 2(c) yields a tip radius of roughly $15 \mathrm{~nm}$, which is in agreement with the value obtained by scanning electron microscopy (SEM) measurements. The origin for this discrepancy is associated with the point dipole model, i.e., the placement of a dipole at the center of a metal sphere of radius $\rho_{\text {tip }}$. However, the dipole model has its limits [13] and a more accurate description of the tip fields requires a shift of the dipole's origin in direction of the tip axis. It is this shift that gives rise to the enlarged value of $\rho_{\text {tip }}=35 \mathrm{~nm}$.

Finally, we emphasize that the theory presented here was developed considering fully coherent scattering. Our choice was based on the fact that the time decay for $\mathrm{co}^{-}$ herent optical phonons in SWNTs is $\tau \sim 1$ ps [14]. Considering typical values of group phonon velocities in SWNTs $\left(v \sim 10^{4} \mathrm{~m} / \mathrm{s}\right)$, the phonon coherence length $\left(L_{\mathrm{ph}}=v \tau\right)$ would be estimated to be $\sim 10 \mathrm{~nm}$. Therefore, it is reasonable to assume that strongly localized light sources such as near-field optical probes mainly detect $\mathrm{co}^{-}$ herent phonons in defect-free SWNTs, since $L_{\mathrm{ph}} \sim \rho_{\text {tip }}$. Nevertheless, the same treatment for incoherent processes is straightforward [9], and our results show that in this case the scattered intensity is inversely proportional to the 11th power of the tip-sample distance. Unfortunately, it was not possible to discern the coherent situation from the incoherent situation experimentally in the present case, since the relations obtained for both cases can satisfactorily fit the experimental data. However, the ongoing fabrication of near-field probes with controlled sizes and shapes [5] will allow one to investigate phonon coherence lengths by means of spatially-resolved Raman spectroscopy, since the value of $\rho_{\text {tip }}$ will be taken as a fix parameter in Eq. (9), and the 10th or 11 th power dependencies can be uniquely determined.

In summary, we have developed a theory describing the near-field Raman enhancement of carbon nanotubes, and more generally, of one-dimensional systems. The theory predicts that the signal enhancement caused by the pres- ence of the gold tip is inversely proportional to the 10th power of the tip-sample distance, and also that the signal is enhanced by roughly the fourth power of the enhancement factor $f_{e}$. We have tested our theory on different carbon nanotubes and found that for defect-free samples the nearfield enhancement does not depend significantly on the specific Raman mode, but mainly on the field enhancement factor $f_{e}$ provided by the near-field probe used in the experiment. It should be noted that the theory outlined here can be easily extended to systems of other dimensionality, such as OD (e.g., molecules or quantum dots), 2D (e.g., graphene or quantum wells), and 3D (e.g., bulk materials).

This work was financially supported by the U.S. Department of Energy (Grant No. DE-FG02-05ER46207). E. J. acknowledges support from the Israel Science Foundation and the U.S.-Israel Binational Science Foundation. A. J. acknowledges Rede de Nanotecnologia CNPq-MCT for financing his visit to Rochester.

*http://www.nano-optics.org

[1] I. O. Maciel, N. Anderson, M. A. Pimenta, A. Hartschuh, H. Qian, M. Terrones, H. Terrones, J. Campos-delgado, A. M. Rao, L. Novotny, and A. Jorio, Nature Mater. 7, 878 (2008).

[2] A. Hartschuh, Angew. Chem., Int. Ed. 47, 8178 (2008).

[3] S. Kawata, Y. Inouye, and P. Verma, Nat. Photon. 3, 388 (2009).

[4] L. Novotny, Prog. Opt. 50, 137 (2007).

[5] Tip Enhancement, Advances in Nano-Optics and NanoPhotonics, edited by S. Kawata and V.M. Shalaev (Elsevier, Amsterdam, 2007).

[6] L. Novotny and S. J. Stranick, Annu. Rev. Phys. Chem. 57, 303 (2006)

[7] M. S. Dresselhaus, G. Dresselhaus, R. Saito, and A. Jorio, Phys. Rep. 409, 47 (2005).

[8] L. X. Benedict, S. G. Louie, and M.L. Cohen, Phys. Rev. B 52, 8541 (1995)

[9] See EPAPS Document No. E-PRLTAO-103-016946 for discussion about the near-field component of the freespace dyadic Green's Function $\overleftrightarrow{\mathbf{G}}^{o}$. This EPAPS also contains information about the coherent properties of the dipole distribution $\mathbf{p}\left(z^{\prime}, \omega_{s}\right)$ in Eq. (6). For more information on EPAPS, see http://www.aip.org/pubservs/epaps.html.

[10] L. Novotny and B. Hecht, Principles of Nano-Optics (Cambridge University Press, New York, 2006).

[11] In the SERS case, the fourth power dependence of the enhancement factor is only valid when the induced Raman dipole is aligned parallel to the electric field [see complete discussion in E.C. Le Ru and P.G. Etchegoin, Chem. Phys. Lett. 423, 63 (2006)].

[12] N. Geblinger, A. Ismach, and E. Joselevich, Nature Nanotech. 3, 195 (2008).

[13] A. Cvitkovic, N. Ocelic, and R. Hillenbrand, Opt. Express 15, 8550 (2007).

[14] D. Song, F. Wang, G. Dukovic, M. Zheng, E. D. Semke, L. E. Brus, and T. F. Heinz, Phys. Rev. Lett. 100, 225503 (2008). 